\title{
A COUPLED BISTABLE STRUCTURE FOR BROADBAND VIBRATION ENERGY HARVESTING
}

\author{
D. Zhu and S.P. Beeby \\ School of Electronics and Computer Science, University of Southampton, Southampton, UK
}

\begin{abstract}
This paper investigates a coupled bistable structure for broadband vibration energy harvesting. The coupled bistable structure consists of two cantilevers with a repelling force between them. By comparing with both conventional bistable and linear structures, it was found that the coupled bistable structure requires lower excitation force to trigger bistable operation than conventional bistable structures. Vibration energy harvesters with coupled bistable structures will have potentially more output power under white noise vibrations, especially low level of vibrations, than both conventional bistable and linear energy harvesters.
\end{abstract}

\section{KEYWORDS}

Broadband vibration energy harvesting, coupled bistable structure.

\section{INTRODUCTION}

Vibration energy harvesters, as a new source of energy, have received increasing levels of attention in the last decade. Mechanical energy from ambient vibrations is converted into electrical energy and this is often an attractive approach for powering wireless sensors. Traditional linear energy harvester produces maximum power when its resonant frequency matches the ambient vibration frequency [1]. Furthermore, to generate maximum power, linear energy harvesters are typically designed to have a high Q-factor. However, in this case, the output power drops dramatically if the ambient and device frequencies do not match. Most reported linear energy harvesters are designed to work only at one fixed resonant frequency [2]. This drawback severely limits the practical applications of vibration energy harvesters.

Methods have been developed to increase operational frequency range of vibration energy harvesting [3] including tuning the resonant frequency of a linear energy harvester [4], using generator array [5], Duffing's nonlinear structures [6] and bistable structures [7]. Tuning the resonant frequency of linear energy harvesters to match the ambient vibration frequency requires energy to be accumulated for the tuning operation and therefore is not suitable for applications where vibration frequency changes rapidly. A generator array consists of an array of linear generators, each of which works at a different frequency. Thus, the assembled generator has a wide operational frequency range. However, at one particular source frequency, only a single or a few individual generators contribute to the power output so the power density of this approach. For a Duffing's nonlinear structure, performance and behaviour depends upon the type of non-linearity (soft or hard spring) and the degree of hysteresis present. The influence of these factors will depend upon whether the driving frequency is increasing or falling. Duffing's nonlinear structures are also prone to instability when operated in the region of hysteresis and output levels can suddenly drop as the structure switches to a lower amplitude state [6]. The bistable structure is a special type of nonlinear structures that has two potential wells. It can be realised with a pre-stressed structure or by applying an external nonlinear force to a linear structure. The existence of the two potential wells allows the inertial mass in the system to travel rapidly between the two equilibrium positions under external mechanical vibrations regardless of their frequencies. Conventional bistable structures have been found to have broader operating frequency ranges than linear structures and behave better than linear structures under white noise excitation [7].

This paper investigates a coupled bistable structure that consists of two cantilevers with a repelling force in-between and its behaviour compared to the conventional bistable structure.

\section{COUPLED BISTABLE STRUCTURE}

A conventional bistable structure as shown in Figure 1(a) consists of a cantilever structure with magnets attached to its free end and other magnets fixed axially in line with the cantilever. The two magnet sets are separated by a distance, $d$, and oriented so that there is a repelling force in-between. The repelling force between the two sets of magnets creates two potential wells and the cantilever jumps between the two equilibrium positions to form a bistable operation.
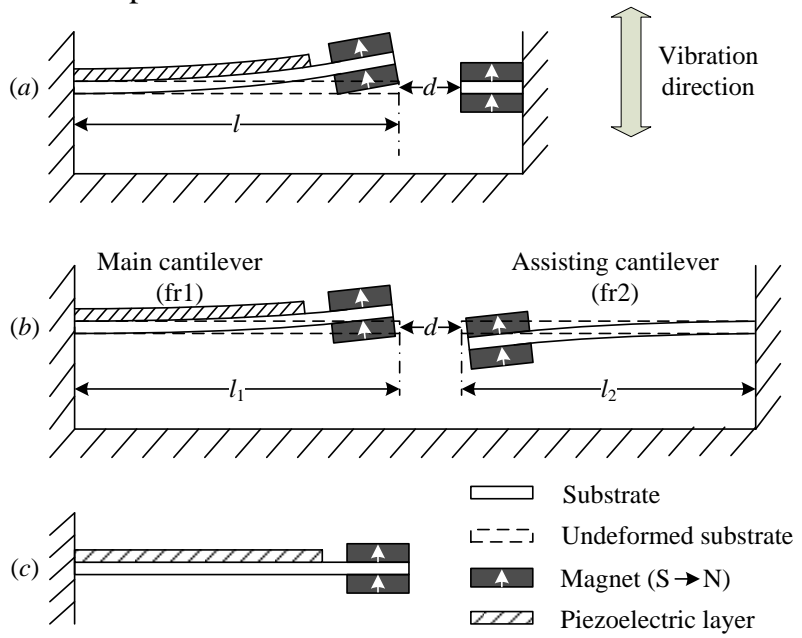

Figure 1: (a) Conventional bistable structure (b) Coupled bistable structure (c) Linear structure.

The proposed coupled bistable structure as shown in Fig. 1(b) consists of two cantilevers, the main cantilever and the assisting cantilever. Two identical magnet sets are placed at free ends of the two cantilevers to produce a repelling force in-between. In the conventional bistable 
structure one magnet is fixed, whereas in the coupled bistable structure both sets of magnet sets are fee to move in the direction of the vibrations. In this case, the behaviour of the main cantilever can be adjusted by varying the characteristics of the assisting cantilever and there are more options available for tuning this behaviour.

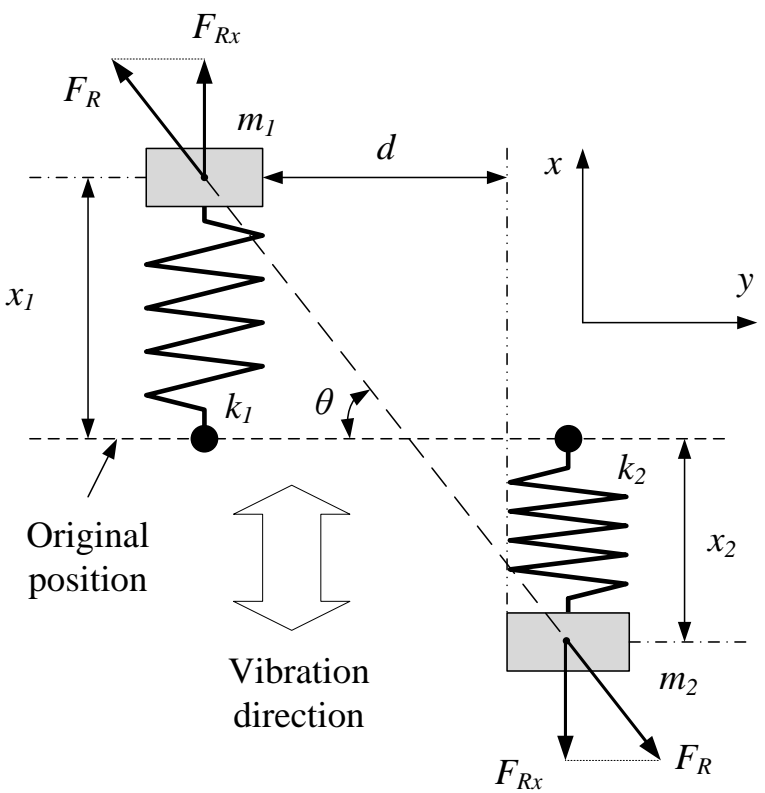

Figure 2: Equivalent model for the coupled bistable structure.

Figure 2 shows an equivalent model of the coupled bistable structure. The main cantilever has a spring constant of $k_{l}$ and an inertial mass (magnet), $m_{l}$ and the assisting cantilever has a spring constant of $k_{2}$ and an inertial mass (magnet), $m_{2} . m_{1}$ and $m_{2}$ are identical in this case. The effect of the gravity is neglected because of the small mass. The repelling force between $m_{l}$ and $m_{2}$ is $F_{R}$ and the its component in the vibration direction, $x$, is $F_{R x}=F_{R} \sin \theta$. The force $F_{R x}$ causes the main and assisting cantilevers to have displacements of $x_{l}$ and $x_{2}$, respectively. Therefore,

$$
k_{1} x_{1}=k_{2} x_{2}=F_{R x}=F_{R} \sin \theta
$$

Thus,

$$
\frac{x_{1}}{x_{2}}=\frac{k_{2}}{k_{1}}
$$

For a constant gap between the two magnet sets, $d$, the overall repelling force, $F_{R}$, is constant. Therefore, the sum of $x_{1}$ and $x_{2}$ is also constant as:

$$
x_{1}+x_{2}=x_{T}
$$

Substation of Eq. 3 into Eq. 2 results in

$$
x_{1}=\frac{k_{2}}{k_{1}+k_{2}} x_{T}
$$

It is found from Eq. 4 that the lower the spring constant (i.e. the lower resonant frequency) of the assisting cantilever, $k_{2}$, the smaller the displacement of the main cantilever, $x_{l}$. This means that the inertial mass on the main cantilever needs to travel less to jump from one equilibrium position to the other. In addition, under mechanical excitation, the two inertial masses, $m_{1}$ and $m_{2}$, travel toward each other, which reduces the excitation force required to trigger bistable operation.

In the extreme cases when $k_{2}=+\infty, m_{2}$ is immobilised and this becomes the conventional bistable structure. When $k_{2}=0, m_{2}$ has unlimited displacement and this becomes the linear structure.

\section{EXPERIMENTAL}

The main cantilever in the coupled bistable structure is made of $\mathrm{BeCu}$ with dimensions of $53 \times 18 \times 0.3 \mathrm{~mm}^{3}$. A Macro Fiber Composite (MFC) piece with dimensions of $35 \times 18 \times 0.2 \mathrm{~mm}^{3}$ was attached to the cantilever to sense its displacement. Two NdFeB magnets with dimensions of $15 \times 15 \times 1 \mathrm{~mm}^{3}$ were fixed to the free end of the cantilever. The resonant frequency of this cantilever, fr 1 , was $26.5 \mathrm{~Hz}$.

The assisting cantilever in the coupled bistable structure is also made of $\mathrm{BeCu}$ and is $18 \mathrm{~mm}$ wide and $0.3 \mathrm{~mm}$ thick. Its length, $l_{2}$, was adjustable so that its resonant frequency, fr2, can be changed. Identical magnets as mentioned above were attached to the free end of the cantilever. Assisting cantilevers of three resonant frequencies were tested, i.e. fr $2=17.3 \mathrm{~Hz}$ when $l_{2}=69$ $\mathrm{mm}$, fr2 $=26.5 \mathrm{~Hz}$ when $l_{2}=58 \mathrm{~mm}$ and fr $2=38.8 \mathrm{~Hz}$ when $l_{2}=46 \mathrm{~mm}$. A conventional bistable structure was made when $l_{2}=0$ and a linear structure was made when the assisting cantilever was removed.

Figure 3 shows the test setup. The two cantilevers sat on a rail that was fixed to a shaker. The distance between the two sets of magnets, $d$, and thus the repelling force in-between can be adjusted. Smaller $d$ means large force. All structures were excited at white noise vibrations with various average accelerations from $2 \mathrm{mG}$ to $25 \mathrm{mG}(1 \mathrm{G}=$ $\left.9.81 \mathrm{~m} \cdot \mathrm{s}^{-2}\right)$. Average output voltage of the MFC piece was recorded for comparisons.

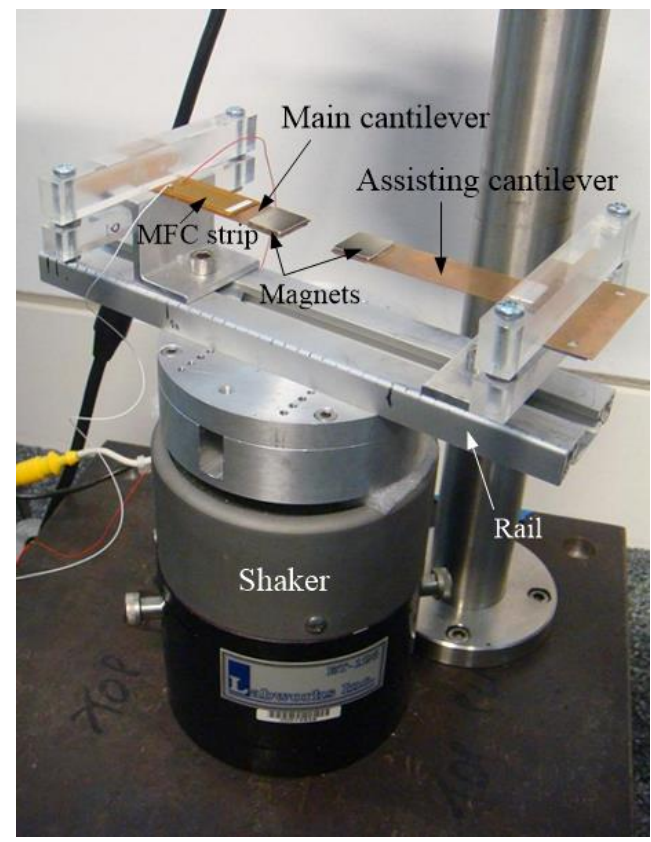

Figure 3: Test setup. 


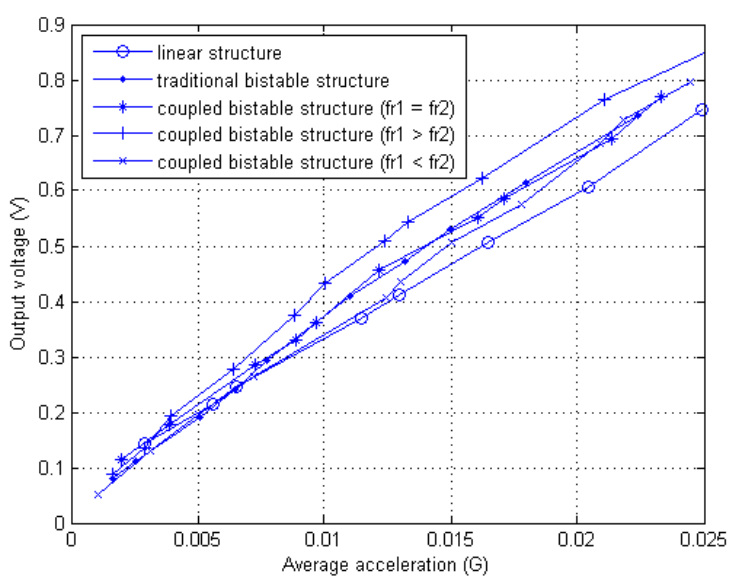

Figure 4: Comparisons of output voltage in different structures when $d=20 \mathrm{~mm}$.

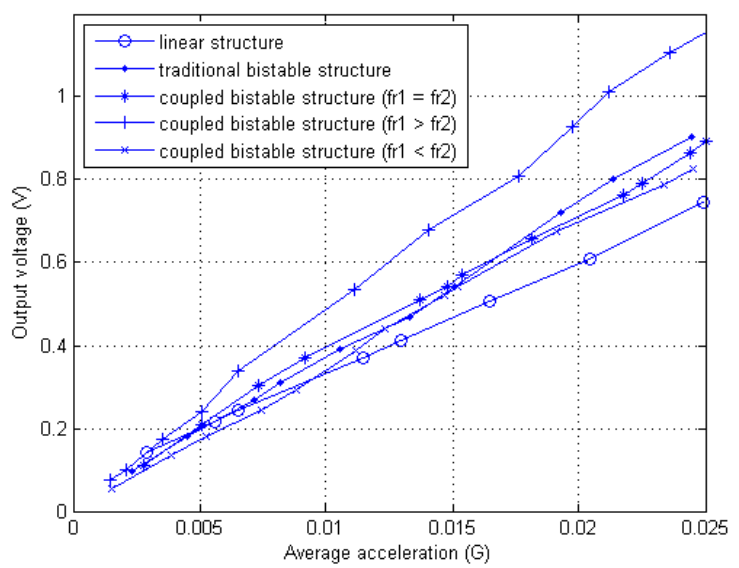

Figure 6: Comparisons of output voltage in different structures when $d=10 \mathrm{~mm}$.

Figures 4 to 7 compare the output voltage in different structures for four different distances between the two sets of magnets, $d$. It was found that the bistable phenomenon occurred in the case of the coupled bistable structure with lower fr 2 for all four situations and the output voltage in this case is always the highest compared to other structures for the same $d$. Bistable phenomenon hardly occurred in other cases except for the coupled bistable structure with equal fr 2 when $d=15 \mathrm{~mm}$.

It was also found that when the average vibration level was lower than $7 \mathrm{mG}$, the linear structure had higher output voltage than the conventional bistable structure and the coupled bistable structure with a higher fr2. This is because that the excitation force is lower than the damping force caused by the repelling magnetic force in bistable structures, which hinders the movement of the cantilever.

Output voltages in all bistable structures are higher than the linear structure when the average vibration level was higher. The only exception was when $d=5 \mathrm{~mm}$ and the output voltage in the linear structure is higher than that in the conventional bistable structure. This is because the repelling force is so large in this situation that the cantilever in the conventional bistable structure is subject to a large pre-stress. Such stress heavily damps the structure and results in lower displacement. In the coupled bistable structure, the pre-stress is much lower because both cantilevers are deflected in the vibration direction and

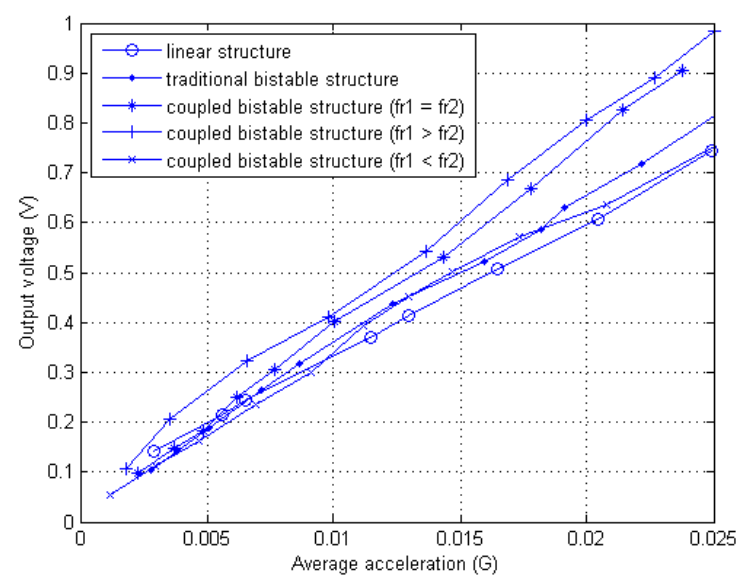

Figure 5: Comparisons of output voltage in different structures when $d=15 \mathrm{~mm}$.

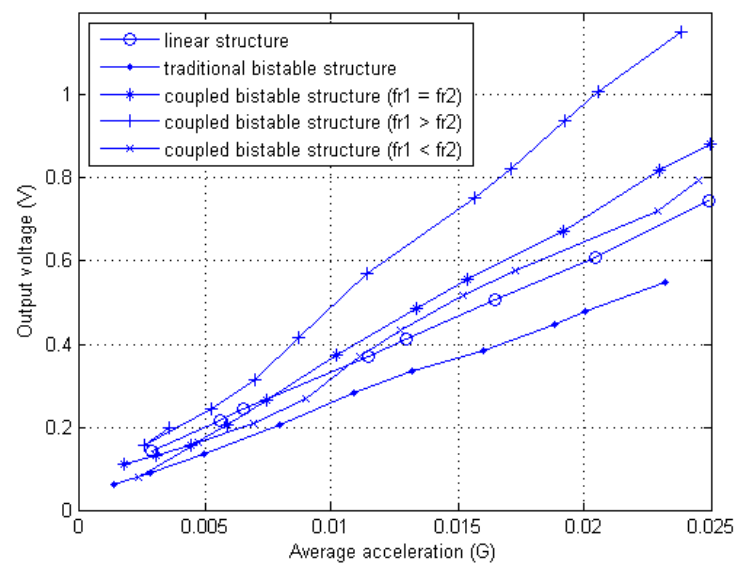

Figure 7: Comparisons of output voltage in different structures when $d=5 \mathrm{~mm}$.

their displacements are much smaller than that of the conventional bistable structure under the same repelling force.

Figures 8 to 11 show the waveform of the five structures when the distance between magnets is $15 \mathrm{~mm}$ under the vibration of an average acceleration of $5 \mathrm{mG}$. It was found that under such low vibrations, the coupled bistable structure with a lower fr2 was already in the bistable mode and thus had the highest output voltage. The coupled bistable structure with an equal fr2 was in the

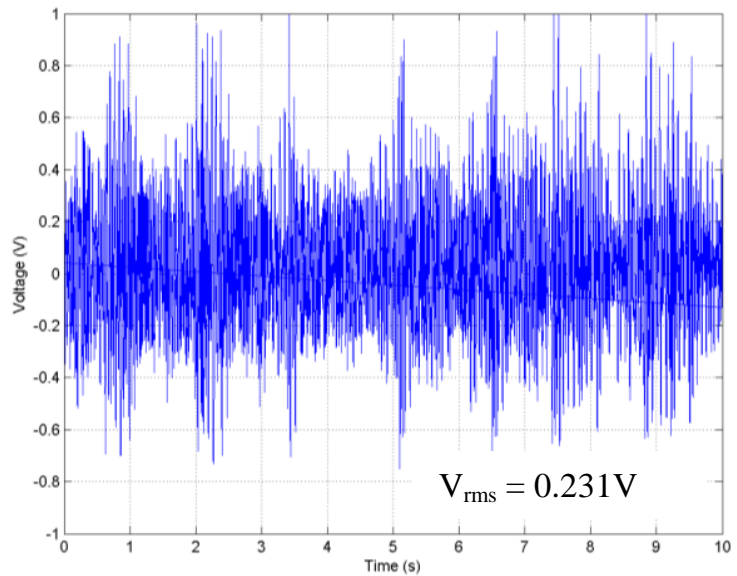

Figure 8: Waveform of the output voltage when frl > fr2 and $d=15 \mathrm{~mm}$ (average acceleration level: $5 \mathrm{mG}$ ). 


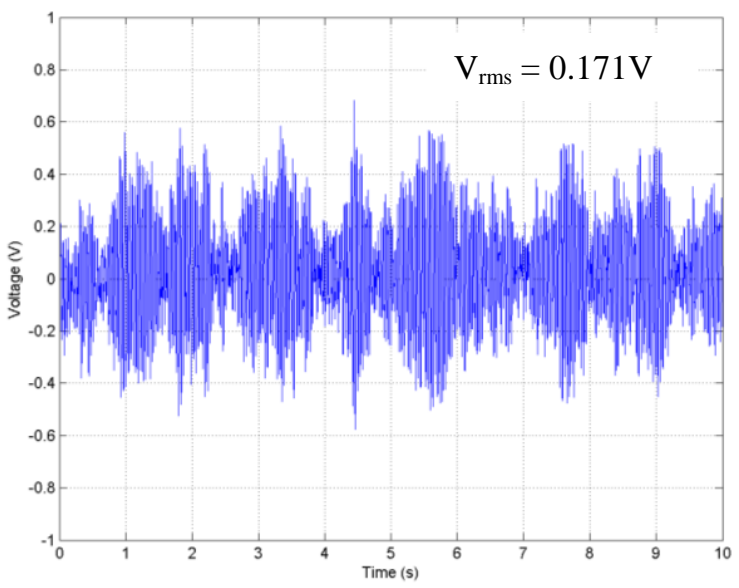

Figure 9: Waveform of the output voltage when fr1 $=$ fr2 and $d=15 \mathrm{~mm}$ (average acceleration level: $5 \mathrm{mG}$ ).

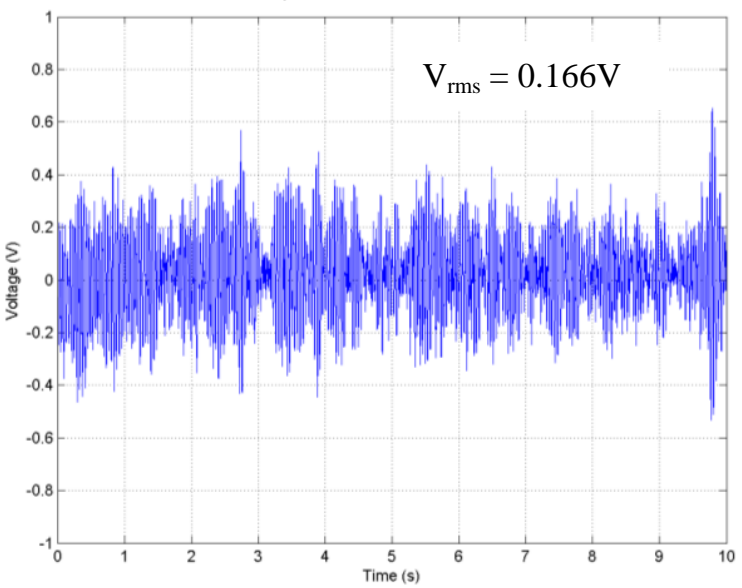

Figure 11: Waveform of the output voltage of the conventional bistable structure when $d=15 \mathrm{~mm}$ (average acceleration level: $5 m G$ ).

mode where the main cantilever oscillated around one equilibrium position. The movement of the main cantilever in the coupled bistable structure with a higher fr2 was hindered due to the reason discussed earlier and thus has the lowest output voltage. The outputs of the conventional bistable and the linear structure were similar under such low vibration as no bistable operation was triggered.

\section{CONCLUSIONS}

It is concluded that a coupled bistable structure is superior to the conventional bistable structure because it requires lower excitation to trigger the bistable operation. It is preferred that the resonant frequency of the assisting cantilever is lower than that of the main cantilever. Therefore, vibration energy harvesters with coupled bistable structures will have potentially more output power under white noise than both conventional bistable and linear energy harvesters. The implementation of the coupled bistable structure in vibration energy harvesters will be investigated in future research.

\section{REFERENCES}

[1] C. B. Williams, R. B. Yates, "Analysis of a micro-electric generator for microsystems", Sensors and Actuators A, vol. 52, pp. 8-11, 1996.

[2] S. P. Beeby, M. J. Tudor, N. M. White, "Energy

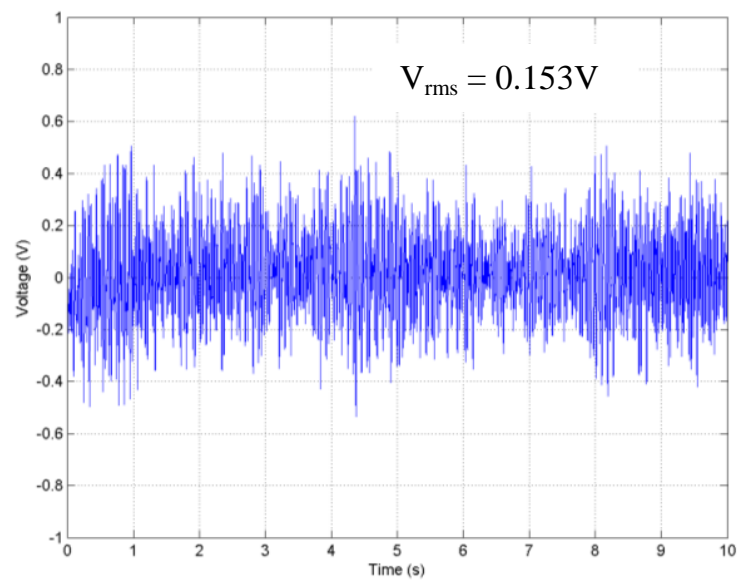

Figure 10: Waveform of the output voltage when frl < fr2 and $d=15 \mathrm{~mm}$ (average acceleration level: $5 \mathrm{mG}$ ).

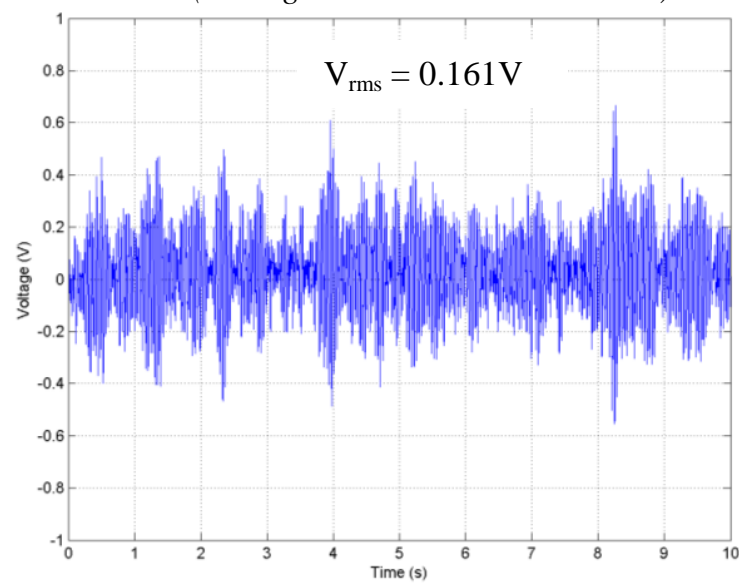

Figure 12: Waveform of the linear structure (average acceleration level: $5 \mathrm{mG}$ ).

harvesting vibration sources for microsystems applications", Meas. Sci. Technol., vol. 17, pp 175195, 2006.

[3] D. Zhu, M. J. Tudor and S. P. Beeby, "Strategies for increasing the operating frequency range of vibration energy harvesters: a review". Meas. Sci. Technol., vol. 21(2), 022001, 2010.

[4] D. Zhu, S. Roberts, J. Tudor and S. P. Beeby, "Design and experimental characterization of a tunable vibration-based electromagnetic micro-generator." Sens. Actua. A, vol. 158(2), pp. 284-293, 2010.

[5] I. Sari I, T. Balkan and H. Kulah, "A wideband electromagnetic micro power generator for wireless microsystems", Int. Solid-State Sensors, Actuators and Microsystems Conf. (Lyon, France) pp 275-278, 2007

[6] B. P. Mann B P and N. D. Sims, "Energy harvesting from the nonlinear oscillations of magnetic levitation", J. Sound Vib., vol. 319, pp. 515-530, 2009.

[7] M. Ferrari, V. Ferrari, M. Guizzetti, B. Andò, S. Baglio, C. Trigona, "Improved Energy Harvesting from Wideband Vibrations by Nonlinear Piezoelectric Converters," Sens. Actua. A, vol. 162. pp. 425-431, 2010.

\section{CONTACT}

*D. Zhu, tel: +44(0)2380595161;dz@ecs.soton.ac.uk 